\title{
Probing vertically graded anisotropy in FePtCu films
}

\author{
Randy K. Dumas, ${ }^{1, *}$ Yeyu Fang, ${ }^{2}$ B. J. Kirby, ${ }^{3}$ Chaolin Zha, ${ }^{2}$ Valentina Bonanni, ${ }^{2}$ Josep Nogués,${ }^{2,4}$ and Johan Åkerman ${ }^{1,2}$ \\ ${ }^{1}$ Department of Physics, University of Gothenburg, Gothenburg 412 96, Sweden \\ ${ }^{2}$ Materials Physics, Royal Institute of Technology (KTH), Kista 164 40, Sweden \\ ${ }^{3}$ Center for Neutron Research, National Institute of Standards and Technology, Gaithersburg, Maryland 20899, USA \\ ${ }^{4}$ Institució Catalana de Recerca i Estudis Avançats (ICREA) and CIN2 (ICN-CSIC) and Universitat Autònoma de Barcelona, \\ Catalan Institute of Nanotechnology, Campus de la Universitat Autònoma de Barcelona, Bellaterra (Barcelona) 08193, Spain
}

(Received 7 January 2011; revised manuscript received 3 June 2011; published 10 August 2011)

\begin{abstract}
Field-dependent polarized neutron reflectivity (PNR) and magnetometry are employed to study the magnetic properties of compositionally uniform and graded $\mathrm{FePtCu}$ films as a function of annealing temperature $\left(T_{A}\right)$. The PNR results are able to directly probe the compositional and anisotropy variations through the film thickness. Further details about how the reversal mechanisms evolve are then elucidated by using a first-order reversal curve technique. The reversal of the graded sample annealed at $300^{\circ} \mathrm{C}$ occurs by an initial rapid switching of the dominant soft A1 phase toward the surface of the film, followed by the gradual reversal of the residual hard phase components toward the bottom. This indicates that the anisotropy gradient is not well established at this low $T_{A}$. A fundamentally different mechanism is found after annealing at $400{ }^{\circ} \mathrm{C}$, where the rapid switching of the entire film is preceded by a gradual reversal of the soft layers. This suggests that the anisotropy gradient has become better established through the film thickness. The field-dependent PNR measurements confirm the existence of an anisotropy gradient, where the lower (higher) anisotropy portions are now toward the bottom (top) of the film because of the $\mathrm{Cu}$ compositional gradient. However, after annealing at $500{ }^{\circ} \mathrm{C}$, a single rapid reversal is found, indicating the formation of a uniform hard film. In this case, PNR demonstrates a more uniform magnetic depth profile that is consistent with a uniform reference sample, suggesting significant interdiffusion of the $\mathrm{Cu}$ is degrading the compositional and induced anisotropy gradient at this elevated $T_{A}$.
\end{abstract}

DOI: 10.1103/PhysRevB.84.054434

PACS number(s): 75.30.Gw, 75.50.Bb, 75.50.Ss

\section{INTRODUCTION}

The study of magnetically heterogeneous systems is a primary focal point of intense research because of their fascinating physical properties and immediate applicability. In particular, binary systems based on ferromagnet $(\mathrm{FM}) /$ antiferromagnet $(\mathrm{AFM})^{1}$ and high anisotropy-FM/low anisotropy-FM ${ }^{2,3}$ layered and nanocomposite materials have garnered the most interest to date. However, graded anisotropy systems, ${ }^{4,5}$ where the anisotropy is continuously varied through the film thickness, are currently being aggressively pursued. Graded anisotropy materials have been primarily motivated by their potential use as magnetic recording media. They join tilted ${ }^{6,7}$ and exchange coupled composite (ECC) media, ${ }^{8}$ as well as heat-assisted magnetic recording ${ }^{9}$ and microwave-assisted magnetic recording ${ }^{10}$ techniques as possible solutions to the magnetic recording "trilemma." 11,12 Successful, long-term storage relies on simultaneously balancing the thermal stability, signal-to-noise ratio, and writability of a given bit. To achieve areal bit densities of in excess of $1 \mathrm{Tbit} / \mathrm{in}^{2}$, the volume of the constituent grains approaches the point where thermal fluctuations begin to dominate as the grains become superparamagnetic. Although high anisotropy materials dramatically improve thermal stability, the magnetic fields required for switching far exceed the capabilities of the write head. Graded anisotropy media is predicted to provide additional gains in writability ${ }^{4,13}$ over conventional bilayer hard/soft ECC media in that the low anisotropy layers act to reduce the switching field while the thermal stability is maintained by the high anisotropy layers. Fabrication schemes to realize graded films remain few and have mostly relied on multilayered approaches. ${ }^{14-19}$
However, a continuous anisotropy gradient has been recently realized in properly annealed, compositionally graded $\mathrm{FePtCu}$ films. ${ }^{20,21}$ In addition, similar FePtCu films have been shown to have a tilted anisotropy ${ }^{22,23}$ that, when combined with the advantages of graded anisotropy, may result in versatile material suitable for both magnetic recording and spin transfer torque applications. ${ }^{24,25}$ Finally, in addition to fabrication challenges, direct experimental verification of an anisotropy gradient is experimentally challenging. Although the inherent depth sensitivity of polarized neutron reflectivity (PNR) measurements ${ }^{17,18}$ have recently been used to directly probe the anisotropy gradient, most experimental works on graded anisotropy films have primarily focused on analysis of the switching field and/or improved thermal stability to indirectly infer the anisotropy gradient.

In this paper, we combine PNR measurements-to directly probe the compositional and anisotropy variations through the film thickness-with the first-order reversal curve (FORC) $)^{21,26-31}$ technique to provide a comprehensive study of how the anisotropy gradient evolves as a function of annealing temperature $\left(T_{A}\right)$ in both compositionally uniform and graded films. In addition to providing a useful qualitative "fingerprint" of the magnetization reversal mechanisms, FORC analysis has shown the ability to extract a wealth of quantitative information in a variety of bulk, ${ }^{32}$ thin film, ${ }^{26,27,33,34}$ and patterned $^{28,30,31,35,36}$ material systems that are not readily accessible from standard major loop or remanence curve analysis. As the $T_{A}$ is increased, distinct changes in the magnetic depth profiles and FORC "fingerprints" are observed. The PNR analysis of the compositionally graded sample annealed at $300^{\circ} \mathrm{C}$ indicates that anisotropy increases with 
proximity to the film/substrate interface, consistent with more rapid formation of the $\mathrm{L}_{0}$ phase toward the bottom, $\mathrm{Cu}$-rich region. However, when the $T_{A}$ is increased to $400{ }^{\circ} \mathrm{C}$, a fundamentally different reversal mechanism is revealed, where the rapid switching of the entire film is initiated by the gradual reversal of the soft layers. Here, the PNR results suggest a reversed anisotropy gradient, where the bottom of the film is now relatively softer. This is consistent with a betterestablished anisotropy gradient throughout the film thickness because of an elevated $T_{A}$, and the $\mathrm{Cu}$ concentration gradient, which now acts to soften the bottom regions compared to the surface. Finally, after annealing at $500^{\circ} \mathrm{C}$, a single rapid reversal process, with only a residual hard phase component, is observed, because significant interdiffusion of the $\mathrm{Cu}$ at this elevated $T_{A}$ results in a compositionally uniform film that is virtually indistinguishable from the compositionally uniform reference sample.

\section{EXPERIMENT}

Both the compositionally uniform and the graded samples were deposited at room temperature on thermally oxidized $\mathrm{Si}$ substrates by magnetron sputtering of high purity $\mathrm{Fe}, \mathrm{Pt}$, and $\mathrm{Cu}$ targets in a chamber with a base pressure better than $6 \times 10^{-6} \mathrm{~Pa}$. To improve surface roughness, (111) texture, and to lower the chemical ordering temperature of the $\mathrm{L1}_{0}$ phase, the $\mathrm{FePtCu}$ films were deposited on a bilayer of $\mathrm{Ta}$ $(6 \mathrm{~nm}) / \mathrm{Pt}(3 \mathrm{~nm})^{37}$ and capped with $5 \mathrm{~nm}$ of Ta to prevent oxidation. To achieve a compositional gradient through the sample thickness, the sputtering power of the $\mathrm{Cu}$ gun was continuously varied from 35 to $0 \mathrm{~W}$. The resulting 20-nm-thick $\left(\mathrm{Fe}_{53} \mathrm{Pt}_{47}\right)_{100-x} \mathrm{Cu}_{x}(x=0-30)$ film (referred to as graded $\mathrm{FePtCu})$ is $\mathrm{Cu}$-rich $\left(\mathrm{Fe}_{53} \mathrm{Pt}_{47}\right)_{70} \mathrm{Cu}_{30}$ at the bottom and $\mathrm{Cu}$-free $\mathrm{Fe}_{53} \mathrm{Pt}_{47}$ at the top. As a reference, a compositionally uniform sample (referred to as uniform $\mathrm{FePtCu}$ ) with the same total $\mathrm{Cu}$ content as the graded sample, $\left(\mathrm{Fe}_{53} \mathrm{Pt}_{47}\right)_{85} \mathrm{Cu}_{15}$, was also deposited. The strong dependence of the A1 (low anisotropy) $\rightarrow \mathrm{L}_{0}$ (high anisotropy) ordering temperature on the $\mathrm{Cu}$ content ${ }^{38}$ is then used to generate an anisotropy gradient in the compositionally graded film. The $\mathrm{Cu}$-rich regions toward the bottom are expected to transform from the as-deposited low anisotropy cubic A1 phase into the high anisotropy tetragonal $\mathrm{L}_{0}$ phase at a lower $T_{A}$ than the $\mathrm{Cu}$-poor regions toward the top, thus establishing the anisotropy gradient. Separate pieces of the graded and uniform $\mathrm{FePtCu}$ films were then annealed at $T_{A}=300{ }^{\circ} \mathrm{C}, 400{ }^{\circ} \mathrm{C}$, and $500{ }^{\circ} \mathrm{C}$ for $35 \mathrm{~min}$ in vacuum. For additional details on sample fabrication and more detailed structural characterization, e.g., x-ray diffraction, see Ref. 20.

Room-temperature, field-dependent PNR measurements on the compositionally graded and uniform $\mathrm{FePtCu}$ films were conducted using the NG1 Reflectometer at the National Institute of Standards and Technology's Center for Neutron Research. A 0.475-nm cold neutron beam was polarized to be spin-up or spin-down relative to an applied magnetic field and was incident on a given sample. The spin-dependent specular reflectivities were measured as functions of the wavevector transfer along the surface normal $\left(Q_{z}\right)$. No significant spin-flip scattering (which arises from the in-plane component of the sample magnetization perpendicular to the applied field) was detected for any sample under any condition; thus, we refer only to the spin-down $(-)$ and spin-up $(+)$ non-spin-flip reflectivities.

The reversal mechanisms have been measured using standard magnetometry with an alternating gradient magnetometer (AGM) at room temperature and with the field applied parallel to the film plane. In addition to standard major loop measurements, the AGM is used to measure families of FORCs using the following procedure. After positive saturation, the applied field is reduced to a given reversal field $H_{R}$. From this reversal field, the magnetization is then measured back toward positive saturation, thereby tracing out a single FORC. This process is repeated for a series of decreasing reversal fields, thus filling the interior of the major hysteresis loop, which can be seen as the outer boundary of the family of FORCs. The FORC distribution is then defined as a mixed second-order derivative of the normalized magnetization,

$$
\rho\left(H, H_{R}\right) \equiv-\frac{1}{2} \frac{\partial^{2} M\left(H, H_{R}\right) / M_{S}}{\partial H \partial H_{R}},
$$

which is then plotted against $\left(H, H_{R}\right)$ coordinates on a contour map. For a given $H_{R}$, the magnetization is measured for increasing applied fields $H$; therefore, $H \geqslant H_{R}$ by design. Following the measurement procedure, the FORC distribution is read in a "top-down" fashion and from left to right for a particular reversal field. The FORC distribution provides a useful fingerprint of the reversal mechanism by mapping out, in $\left(H, H_{R}\right)$ coordinates, only the irreversible switching processes. It is often useful to have a one-dimensional visualization of the irreversibility by projecting the FORC distribution onto the $H_{R}$ axis. This is equivalent to an integration over the applied field $H$,

$$
\int \frac{\partial^{2} M\left(H, H_{R}\right)}{\partial H \partial H_{R}} d H=\frac{\partial M\left(H_{R}\right)}{\partial H_{R}},
$$

and is termed a FORC-switching field distribution (FORCSFD). This can then be easily compared with the standard technique of taking the derivative of the dc-demagnetization (DCD) curve to determine the DCD-switching field distribution (DCD-SFD):

$$
\frac{\partial M_{r}\left(H_{R}\right)}{\partial H_{R}} \text {. }
$$

Here $M_{r}\left(H_{R}\right)$ is the zero-field magnetization, or remanence, after the application of a given reversal field $H_{R}$. The DCDSFD data can be conveniently extracted from the measured family of FORCs as the remanent state is measured for each reversal curve. For direct comparison, both the FORC-SFD and the DCD-SFD are normalized by their maximum value. Although the DCD-SFD and FORC-SFD are similar, they are sensitive to different aspects of the reversal. The DCD-SFD only probes switching processes as manifested at the remanent state, whereas the FORC-SFD accounts for irreversibility along the entire reversal curve.

\section{RESULTS AND DISCUSSIONS}

The inherent sensitivity to the nuclear and magnetic depth profiles $^{39,40}$ makes PNR an ideal probe for graded anisotropy thin films. ${ }^{17,18}$ PNR measurements demonstrate strong 


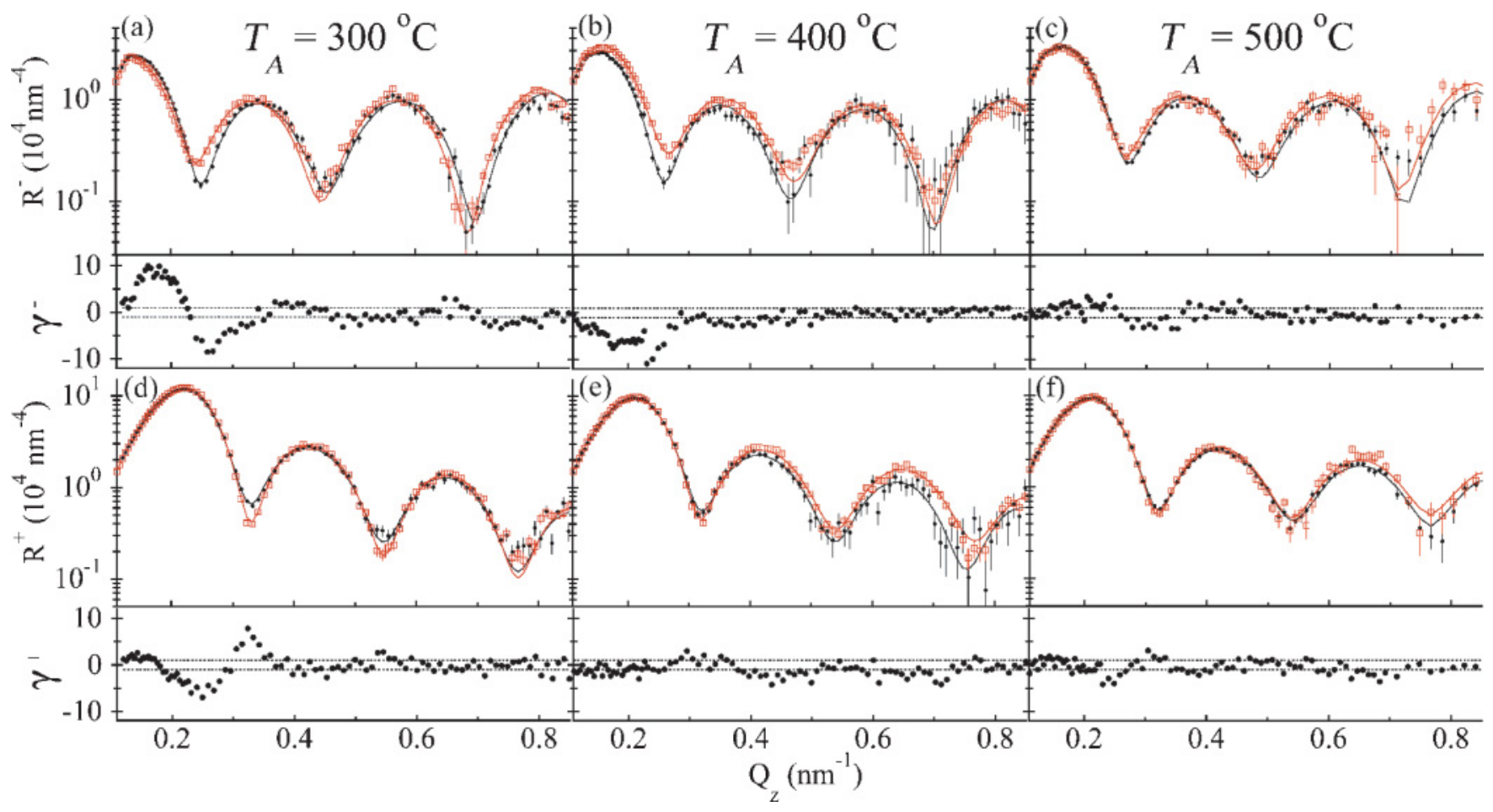

FIG. 1. (Color online) Fitted spin-down $R^{-}$(a-c) and spin-up $R^{+}(\mathrm{d}-\mathrm{f})$ reflectivities for the graded (black online) and uniform (red online) samples. The uncertainty-normalized difference in the data $\gamma$ is shown along the bottom of each panel. Dashed lines in the $\gamma$ plots correspond to \pm 1 . Measurements were taken in $750 \mathrm{mT}$ for $T_{A}=300^{\circ} \mathrm{C}$ (saturation, a, d), $820 \mathrm{mT}$ for $T_{A}=400{ }^{\circ} \mathrm{C}$ (quasisaturation, b, e), and $1 \mathrm{mT}$ for $T_{A}=500^{\circ} \mathrm{C}$ (remanence, c, f). Error bars correspond to $\pm 1 \sigma$.

sensitivity to $\mathrm{Cu}$ gradation in the samples, as evidenced by Fig. 1, which shows a comparison of PNR spectra for uniform and graded samples at fields corresponding to essentially flat regions of the hysteresis loop: a $750 \mathrm{mT}$ saturating field for $T_{A}=300^{\circ} \mathrm{C}$, an $820 \mathrm{mT}$ quasisaturating field (coming up from $-850 \mathrm{mT}$ ) for $T_{A}=400{ }^{\circ} \mathrm{C}$, and a remanent $1 \mathrm{mT}$ field (after saturating offline in $1.6 \mathrm{~T}$ ) for $T_{A}=500^{\circ} \mathrm{C}$. To better visualize the data at all $Q_{z}$, the fitted spin-down $R^{-}$(Figs. 1(a)-1(c)) and spin-up $R^{+}$(Figs. 1(d)-1(f)) reflectivities are shown multiplied by $Q_{z}{ }^{4}$ (the approximate reflectivity falloff for a perfect interface). Each Fig. 1 column corresponds to a different $T_{A}$ and shows fitted data for the graded $\left(R_{G}\right)$ and uniform $\left(R_{U}\right)$ samples, with the residual difference in the data normalized by the experimental uncertainty $\gamma=\frac{R_{G}-R_{U}}{\sqrt{\mathrm{d} R_{G}^{2}+\mathrm{d} R_{U}^{2}}}$, shown along the bottom of each panel. Normalized in this way, values of $|\gamma|>1$ indicate statistically significant differences between graded and uniform samples. Figure 1 shows that the PNR spectra for the graded/uniform pairs become more similar at a high $T_{A}$ - c clear indication that elevated $T_{A}$ results in similar depth profiles for the two types of samples. This qualitative assessment is borne out by the models used to fit the data, which are shown in Fig. 2. Using fully dynamical calculations, ${ }^{39,40}$ the data were well fit in terms of nuclear scattering length density $\rho(z)$ (Figs. 2(a)-2(c), indicative of the nuclear composition) and in-plane magnetization $M(z)$ (Figs. 2(d)-2(f)). ${ }^{41}$ The magnetizations of the $\mathrm{FePtCu}$ films were assumed to vary linearly with distance from the $\mathrm{SiO}_{2}$ interface $z$, whereas the cap magnetization was allowed to vary freely. The effects of $\mathrm{Cu}$ gradation are clearly manifested in the magnetic profiles (Figs. 2(d)-2(f)). For all three $T_{A}$, the graded samples exhibit a clear reduction in magnetization with decreasing $z$, originating from a progressive reduction in the volume fraction of $\mathrm{Fe}$ as the $\mathrm{Cu}$ concentration is increased. ${ }^{42}$ In stark contrast, the uniform reference samples show significantly flatter magnetization profiles, consistent with more uniform $\mathrm{Cu}$ profiles. $T_{A}$ is observed to have a significant effect on the graded sample, because the $T_{A}=$ $300^{\circ} \mathrm{C}$ and $T_{A}=400^{\circ} \mathrm{C}$ graded FePtCu films exhibit a roughly $25 \%$ decrease in magnetization from top to bottom, whereas the effect is $\sim 15 \%$ for the $T_{A}=500{ }^{\circ} \mathrm{C}$ film. So, as suggested by the data comparison in Fig. 1, model fitting confirms that the parts of the $T_{A}=500^{\circ} \mathrm{C}$ graded/uniform pair have more similar magnetic profiles than do the lower $T_{A}$ pairs. The overall decrease in the average magnetization, as $T_{A}$ is increased, is consistent with a greater proportion of the film having transformed into the $\mathrm{L} 1_{0}$ phase. ${ }^{4-45}$ Moreover, the best-fit models for all samples show a nonzero magnetization for the nominally Ta surface capping layer, implying some amount of vertical $\mathrm{Fe}$ diffusion for all $T_{A}$.

In addition, we have studied the field-dependent properties of the differently annealed samples using PNR and FORC techniques. Field-dependent PNR directly probes depth-dependent anisotropy variations, whereas FORC identifies switching events during the reversal process.

\section{A. $T_{A}=300^{\circ} \mathrm{C}$}

Model fitting of PNR data at varying fields (not shown) yields the magnetic profiles shown in Figs. 3(a) and 3(b). The in-plane magnetization depth profile $M(z)$, in absolute units, is 


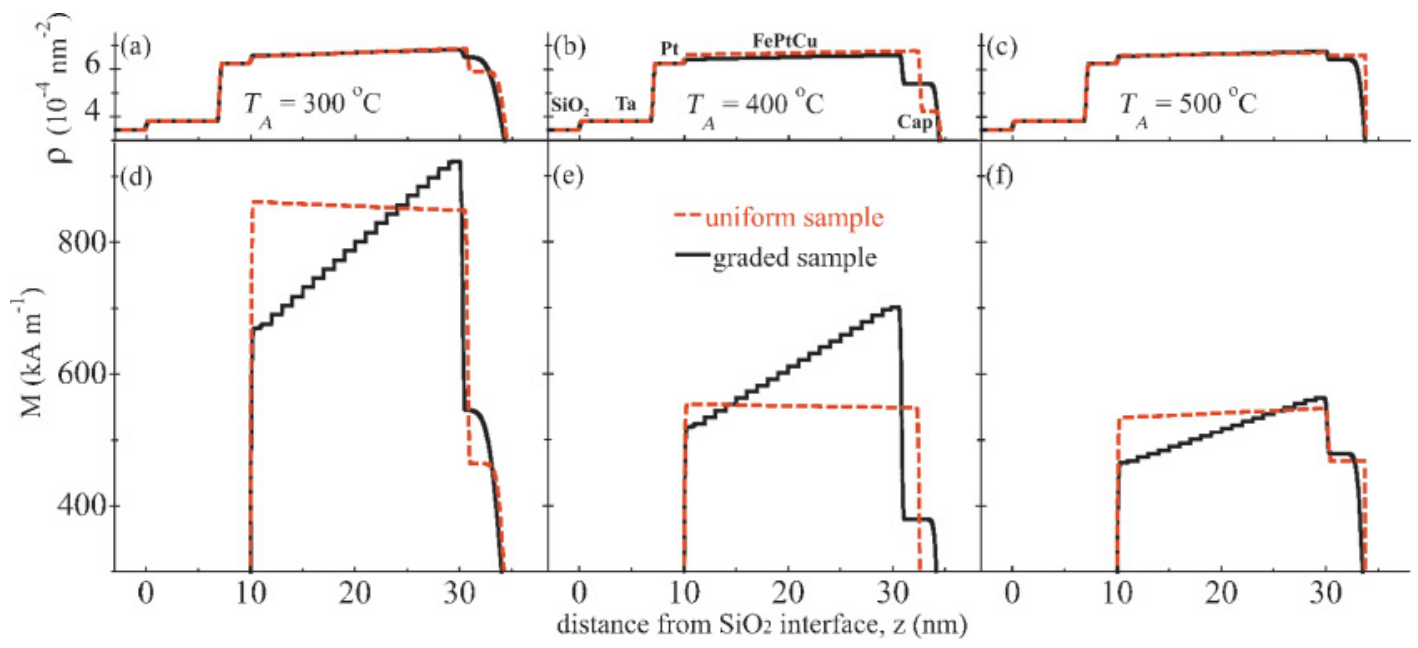

FIG. 2. (Color online) Nuclear (a-c) and magnetic (d-f) profiles used to fit the PNR data shown in Fig. 1.

shown in Fig. 3(a) as a function of applied field after negative saturation in $-750 \mathrm{mT}$. To better distinguish depth variations in anisotropy from depth variations in total moment, the profiles in Fig. 3(a) are normalized by the positive saturation profile, as shown in Fig. 3(b). For a small positive field of $30 \mathrm{mT}$, the $M / M_{\text {sat }}$ profile is essentially flat, because the sample is still nearly negatively saturated. However, as the applied field is increased, dramatic variations in the depth-dependent anisotropy start to become clear. The near surface, $\mathrm{Cu}$-poor region of the $\mathrm{FePtCu}$ film approaches saturation $\left(M / M_{\text {sat }}=1\right)$ faster than does the $\mathrm{Cu}$-rich bottom, and the Fe-infused Ta cap approaches saturation even faster than that. Clearly, the bottom of the film only approaches saturation in a relatively large field, because this $\mathrm{Cu}$-rich region has preferentially transformed into the high anisotropy $\mathrm{L} 1_{0}$ phase. Thus, the sample exhibits a soft cap and a pronounced vertical anisotropy variation throughout the $\mathrm{FePtCu}$ film, as schematically represented in Fig. 3(c). These results also qualitatively agree with the magneto-optical Kerr effect on much thicker $(50 \mathrm{~nm})$ compositionally graded FePtCu films annealed at $300{ }^{\circ} \mathrm{C}$, where the finite penetration depth of the laser showed the topmost $\sim 30 \mathrm{~nm}$ to be relatively soft. ${ }^{21}$ Constraining the models to have a uniform anisotropy profile results in a significantly worse fit to the data.

Further insight into how the observed depth-dependent anisotropy variation affects the reversal mechanisms is carried out by analyzing the family of FORCs and the corresponding FORC distribution (calculated using Eq.(1)), shown in Figs. 4(a) and 4(b), respectively, for the graded FePtCu film annealed at $300{ }^{\circ} \mathrm{C}$. The major loop, seen as the outer boundary of the FORCs, has a coercivity of $70 \mathrm{mT}$ and exhibits a single sharp switching process. However, the FORC distribution (Fig. 4(b)), which reveals subtler information about the reversal, is characterized by two primary features: a peak located at $\left(H, H_{R}\right)=(10 \mathrm{mT},-70 \mathrm{mT})$, indicated by a (red) dashed circle, and a negative-positive tail that extends downward parallel to the $H_{R}$ axis, indicated by a (black) dashed oval. The peak not only locates, in $\left(H, H_{R}\right)$ coordinates, the maximum irreversibility during switching but also reveals a great deal about the magnetic interactions present within the film. To visualize this, the FORC that originates at $\mu_{o} H_{R}=-70 \mathrm{mT}$ is highlighted with a bold (red) line in Fig. 4(a) and a horizontal (red) dashed line in Fig. 4(b). We see that the highlighted FORC originates from a nearly demagnetized state near the coercive field, where a high degree of irreversible switching would be expected. More interestingly, as the applied field $H$ is increased from $\mu_{o} H_{R}=-70 \mathrm{mT}$, the highlighted FORC in Fig. 4(a) reaches positive saturation in a relatively small field, with a maximum slope at $\mu_{o} H=10 \mathrm{mT}$. If we consider the minor loop bounded by the descending branch of the major loop and the (red) highlighted FORC in Fig. 4(a), we clearly see it is significantly shifted toward negative applied fields. This behavior suggests the dominant soft phase components of the film are ferromagnetically exchange coupled to a higher anisotropy phase. Evidence of this higher anisotropy phase is found in the extended negative-positive tail in Fig. 4(b). This tail is generated by the irreversibility associated with switching the hardest components of the film, which require a more negative reversal field to fully switch. This feature, which extends to reversal fields of nearly $\mu_{o} H_{R}=-600 \mathrm{mT}$, indicates that true saturation only occurs for very large negative reversal fields when the FORC diagram becomes featureless. ${ }^{26}$ This high field saturation is also evident in the PNR data, which clearly show a nonsaturated profile at $120 \mathrm{mT}$ (Fig. 3(b)). The high anisotropy contributions to the reversal also become apparent in the asymmetric FORC-SFD (Eq. (2)) and DCD-SFD (Eq. (3)) in Fig. 4(c), which both show an extended switching tail that extends toward large negative reversal fields. This asymmetry is consistent with the initial rapid switching of the dominant soft phase and gradual reversal of the hard phase components. From a qualitative fingerprinting standpoint, the FORC distribution and SFDs are strikingly similar to that of a previously studied polycrystalline $\mathrm{FePt} / \mathrm{FeNi}$ hard/soft exchange spring bilayer. ${ }^{27}$ In our graded FePtCu films, annealing at $300^{\circ} \mathrm{C}$ has preferentially increased the $\mathrm{L} 1_{0}$ ordering of the $\mathrm{Cu}$-rich portions near the bottom of the film, while most of the film remains in the low anisotropy A1 phase, thus creating a similar, although not as well defined, hard/soft bilayer structure, which is schematically 


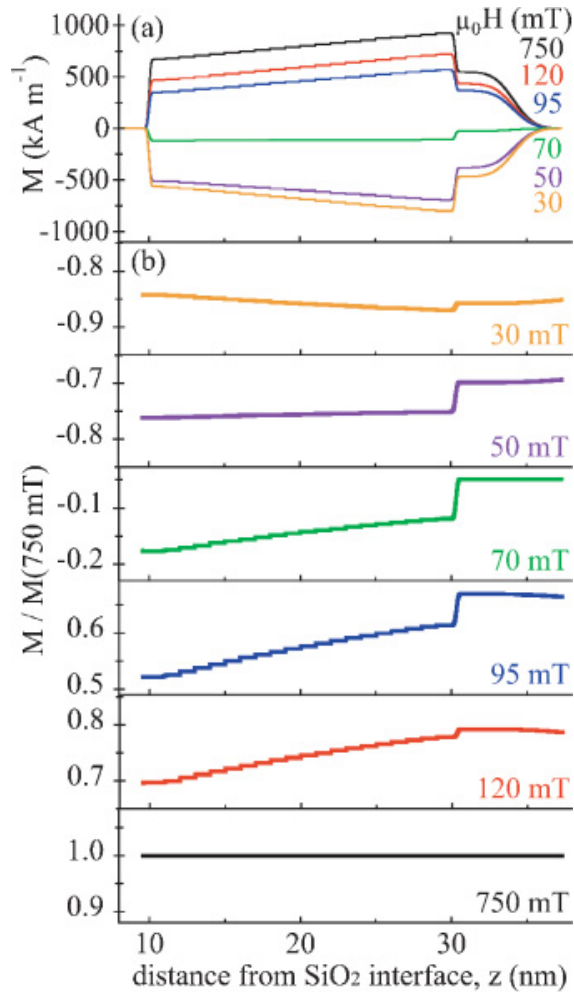

(c)

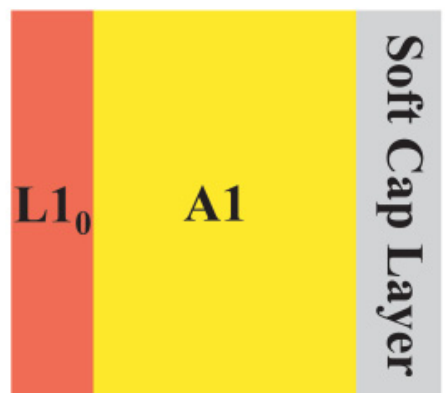

FIG. 3. (Color online) (a) Magnetization profiles used to fit the field-dependent PNR spectra for the graded sample annealed at $300^{\circ} \mathrm{C}$. (b) Normalized magnetization profiles highlighting the anisotropy variation through the film thickness at the indicated fields after negative saturation in $-750 \mathrm{mT}$. (c) Sample schematic highlighting the expected depth dependence of the phase profile.

represented in Fig. 3(c). We therefore conclude that $T_{A}=$ $300{ }^{\circ} \mathrm{C}$, although able to transform a portion of the film into the high anisotropy $\mathrm{L} 1_{0}$ phase, is not sufficient to establish an appropriate anisotropy gradient through the entire film thickness.

As a comparison, measurements on a compositionally uniform $\mathrm{FePtCu}$ film, also annealed at $300^{\circ} \mathrm{C}$, are conducted (Figs. 4(d)-4(f)). The most obvious difference is that the uniform FePtCu sample has a much smaller coercivity, $24 \mathrm{mT}$, as compared to the graded $\mathrm{FePtCu}$ sample. This is expected, because $300{ }^{\circ} \mathrm{C}$ is not sufficient to transform $\left(\mathrm{Fe}_{53} \mathrm{Pt}_{47}\right)_{85} \mathrm{Cu}_{15}$ into the high anisotropy $\mathrm{L} 1_{0}$ phase. In addition, differences in the reversal mechanisms become apparent when comparing FORC distributions of the graded and uniform samples (Figs. 4(b) and 4(e), respectively). Although both clearly

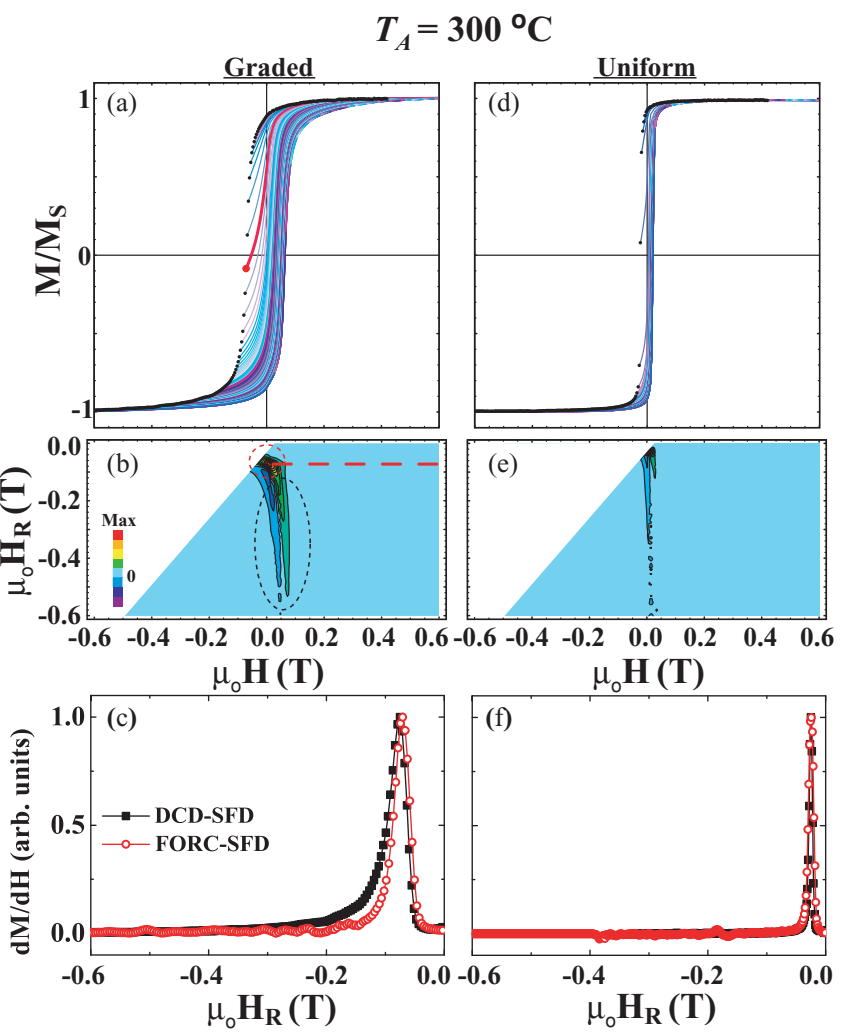

FIG. 4. (Color online) Families of FORCs, FORC distributions, and SFDs for the graded (left) and uniform (right) $\mathrm{FePtCu}$ films annealed at $300^{\circ} \mathrm{C}$. The first point of each reversal curve in (a) and (d) is shown by a black dot; the corresponding FORC distributions, shown as contour plots, are in (b) and (e), respectively. The horizontal (red) dashed line in (b) corresponds to the (red) bold FORC in (a), and the dashed (red) circle and dashed (black) oval highlight regions of the FORC diagram discussed in the text. The DCD-SFD as closed (black) squares and FORC-SFD as open (red) circles for the graded and uniform films are shown in (c) and (f), respectively.

show a sharp irreversible peak, the prominent extended negative-positive tail found in the graded sample is mostly absent in the uniform film. Furthermore, the DCD-SFD and FORC-SFD (Fig. 4(f)) are symmetric and far narrower than the graded samples (Fig. 4(c)). These results indicate that this film primarily consists of a single highly uniform soft phase. However, the faint irreversible tail observed in the FORC distribution (Fig. 4(e)) reveals that even the nominally uniform film shows some small degree of anisotropy variation.

$$
\text { B. } T_{A}=400{ }^{\circ} \mathrm{C}
$$

Interestingly, after annealing at $400{ }^{\circ} \mathrm{C}$, the variable field PNR measurements (measured after applying $-820 \mathrm{mT}$ ), reveal a dramatically different anisotropy profile than what was observed for $T_{A}=300{ }^{\circ} \mathrm{C}$. Figure 5 shows the absolute (a) and normalized (b) field-dependent magnetization depth profiles. The normalized profiles indicate that the surface region again approaches saturation faster than the $\mathrm{FePtCu}$ film, consistent with a soft, Fe-infused Ta cap. More striking however, is that the $\mathrm{Cu}$-rich bottom of the $\mathrm{FePtCu}$ film approaches saturation in a smaller field than the $\mathrm{Cu}$-deficient top-the opposite of 


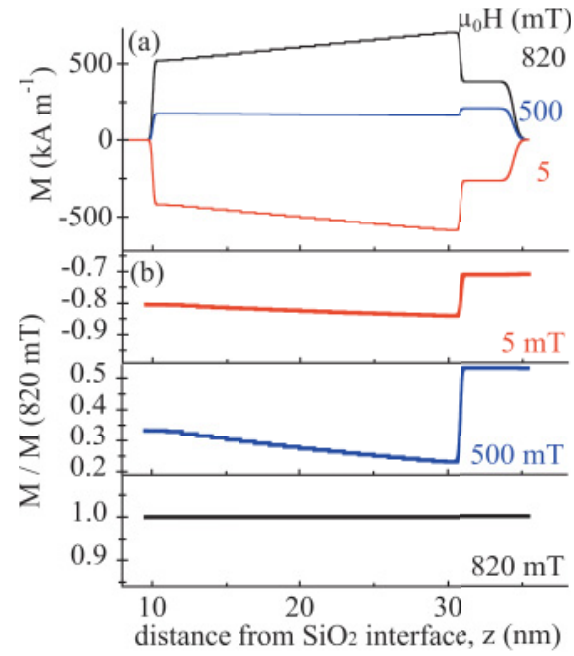

(c)

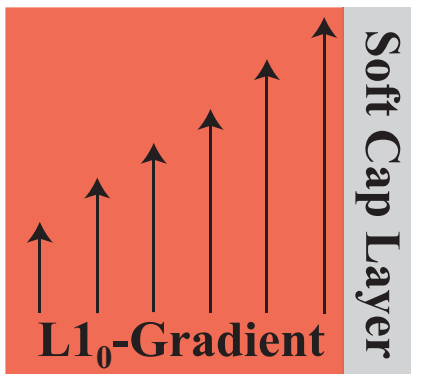

FIG. 5. (Color online) (a) Magnetization profiles used to fit the field-dependent PNR spectra for the graded sample annealed at $400{ }^{\circ} \mathrm{C}$. (b) Normalized magnetization profiles highlighting the anisotropy variation through the film thickness at the indicated fields after negative saturation in $-820 \mathrm{mT}$. (c) Sample schematic highlighting the expected depth dependence of the phase profile, where the length of the black arrows represents the relative strength of the $\mathrm{L} 1_{0}$ phase at that particular depth.

what was observed for the $T_{A}=300^{\circ} \mathrm{C}$ sample. This inverted anisotropy profile has a simple explanation. At this elevated $T_{A}$, the total fraction of the film that is in the high anisotropy $\mathrm{L}_{0}$ phase has increased dramatically, as evident in the increased major loop coercivity of $548 \mathrm{mT}$ (Fig. 6(a)). However, a significant compositional gradient still exists, as evident in Fig. 2(e). So, although the $\mathrm{Cu}$-rich regions near the bottom of the film have undergone the $\mathrm{A} 1 \rightarrow \mathrm{L}_{0}$ transformation at a lower $T_{A}$ as compared to the rest of the film, the relative anisotropy strength is reduced because of the increased $\mathrm{Cu}$ content, ${ }^{42}$ thus establishing a $\mathrm{L}_{0}$ gradient $^{45}$ through the bulk of the film thickness, which is now relatively soft toward the bottom, as schematically represented in Fig. 5(c).

Significant differences in the reversal mechanisms using the FORC technique are also observed after annealing at $400{ }^{\circ} \mathrm{C}$ (Figs. 6(a)-6(c)). Two differences in the major loop (Fig. 6(a)) are immediately apparent from the sample annealed at $300^{\circ} \mathrm{C}$. First, the coercivity has dramatically increased to $548 \mathrm{mT}$ because of the overall increase in $\mathrm{L}_{0}$ ordering. Second, the shape of the major loop is markedly different. Instead of showing a single sharp reversal, a more gradual approach to saturation is observed and the reversal appears to occur in two stages. The FORC distribution (Fig. 6(b)) has also dramatically

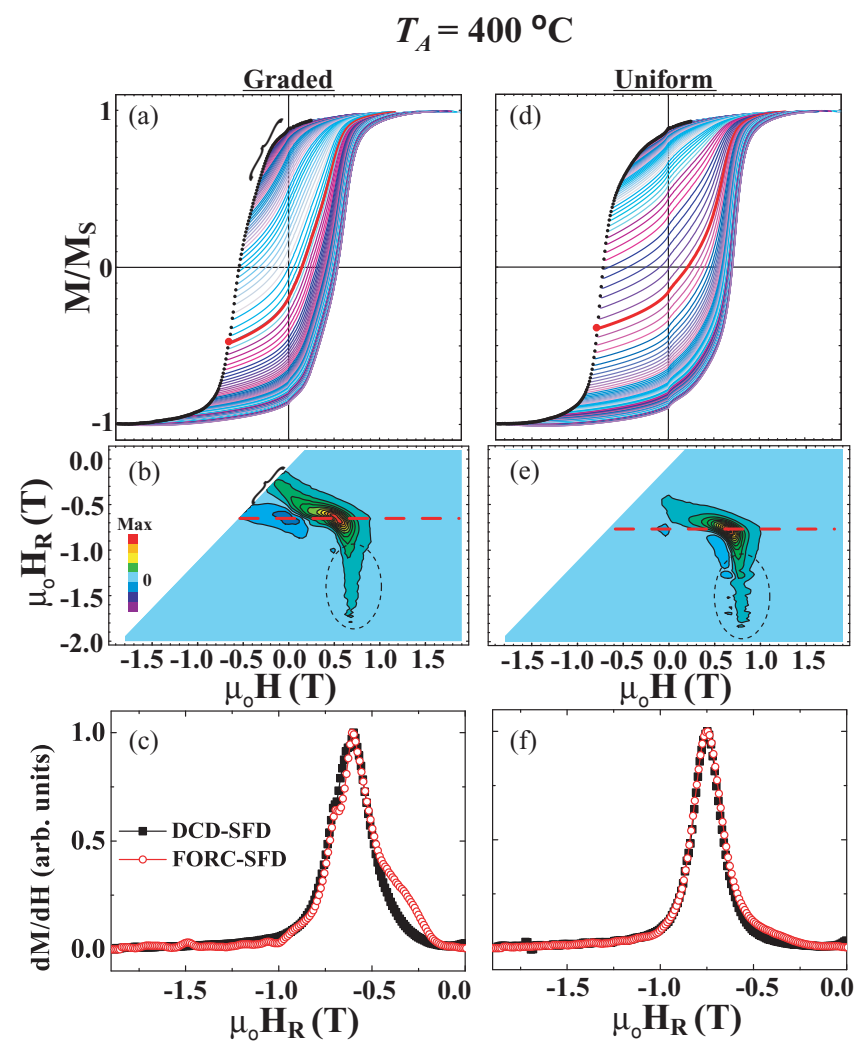

FIG. 6. (Color online) Families of FORCs, FORC distributions, and SFDs for the graded (left) and uniform (right) $\mathrm{FePtCu}$ films annealed at $400{ }^{\circ} \mathrm{C}$. The first point of each reversal curve in (a) and (d) is shown by a black dot and the corresponding FORC distributions, shown as contour plots, are in (b) and (e), respectively. The horizontal (red) dashed lines in (b) and (e) correspond to the (red) bold FORCs in (a) and (d), respectively. The dashed black ovals in (b) and (e) and the brackets in (a) and (b) highlight regions discussed in the text. The DCD-SFD as closed (black) squares and FORC-SFD as open (red) circles for the graded and uniform films are shown in (c) and (f), respectively.

changed from the one observed for $T_{A}=300{ }^{\circ} \mathrm{C}$ (Fig. 4(b)), indicating a fundamentally different reversal mechanism. The FORC diagram is completely featureless for $\mu_{o} H_{R} \geqslant-150$ $\mathrm{mT}$ because of reversible switching processes. Then, for reversal fields of $-550 \mathrm{mT} \leqslant H_{R} \leqslant-150 \mathrm{mT}$, indicated with a bracket in Figs. 6(a) and 6(b), a low intensity ridge develops in the FORC diagram, marking the beginning of irreversible switching processes as the low anisotropy portions of the film begin to reverse. As the reversal field continues to decrease, the maximum irreversibility is found along $\mu_{o} H_{R}=-650 \mathrm{mT}$, where a prominent negative-positive pair of peaks appears in the FORC diagram, indicated with a horizontal (red) dashed line in Fig. 6(b), and corresponds to the rapid irreversible switching of the film. As was similarly done in Fig. 4(a) for the sample annealed at $300^{\circ} \mathrm{C}$, the FORC that corresponds to the maximum irreversibility, at $\mu_{o} H_{R}=-650 \mathrm{mT}$, is highlighted with a bold (red) line in Fig. 6(a) to indicate the reversal path back toward positive saturation. Unlike the behavior exhibited in Fig. 4(a), the highlighted FORC in Fig. 6(a) gradually approaches positive saturation in a highly linear fashion and in a large applied field. This behavior is consistent with an 
anisotropy gradient that has become better established through the film thickness at this $T_{A}$.

The FORC-SFD (Fig. 6(c)) is also fundamentally different from the sample annealed at $300{ }^{\circ} \mathrm{C}$ (Fig. 4(c)). Although both are highly asymmetric, the nature of the asymmetry is opposite for each sample. As the reversal field approaches more negative values, the sample annealed at $300{ }^{\circ} \mathrm{C}$ rapidly rises and then gradually decreases, whereas the sample annealed at $400{ }^{\circ} \mathrm{C}$ shows an initial gradual increase followed by a rapid fall. Because the anisotropy gradient has become better established after annealing at $400{ }^{\circ} \mathrm{C}$, the reversal begins gradually as the low anisotropy layers slowly begin to reverse, in stark contrast to the sample annealed at $300^{\circ} \mathrm{C}$, which was dominated by the initial rapid switching of the dominant soft phase component. During the early stages of reversal, the DCD-SFD and FORCSFD clearly differ (Fig. 6(c)), in that the FORC-SFD shows a significant shoulder that precedes the primary switching peak. This highlights the key difference between the two SFDs. ${ }^{21}$ During the initial soft phase reversal, the remanent state changes little, which in turn renders it virtually invisible in the DCD-SFD. However, because the FORC-SFD is sensitive to the irreversibility associated with the soft phase reversal, it is able to clearly resolve the initial reversal stages. This shoulder in the FORC-SFD can be directly traced to the aforementioned low intensity ridge that developed in the FORC diagram for $-550 \mathrm{mT} \leqslant \mu_{o} H_{R} \leqslant-150 \mathrm{mT}$. Finally, as is also apparent in the FORC distribution, a faint tail, highlighted with a black dashed oval in Fig. 6(b), indicates the irreversibility associated with a residual hard phase component that is difficult to saturate.

Most significantly, the coercivity of the graded $\mathrm{FePtCu}$ sample, $548 \mathrm{mT}$, is considerably smaller than the uniform $\mathrm{FePtCu}$ reference sample, $730 \mathrm{mT}$, also annealed at $400^{\circ} \mathrm{C}$. This is expected, because a properly induced anisotropy gradient should result in a smaller switching field. The reversal mechanism of the uniform $\mathrm{FePtCu}$ sample is also markedly different. The (red) highlighted FORC in Fig. 6(d), which originates at $\mu_{o} H_{R}=-779 \mathrm{mT}$ and intersects the primary peak in the FORC distribution (Fig. 6(e)), approaches saturation differently than the graded sample (Fig. 6(a)). Whereas the graded sample is highly linear during much of its approach to saturation, the uniform sample is more concave. These subtle differences become clear in the FORC distributions. From a simple fingerprinting standpoint, the FORC distribution (Fig. 6(e)) indicates that the reversal mechanism of the uniform FePtCu sample is qualitatively similar to the uniform $\mathrm{FePtCu}$ sample annealed at $300{ }^{\circ} \mathrm{C}$ (Fig. 4(e)). Both show a single peak followed by a weak high anisotropy tail, indicated with a black dashed oval. In addition, both the DCD-SFD and the FORC-SFD (Fig. 6(f)) show a single highly symmetric peak and virtually overlap, as was also observed in Fig. 4(f). Interestingly, much like the uniform $\mathrm{FePtCu}$ sample annealed at $300^{\circ} \mathrm{C}$, the irreversible tail, indicated with the dashed black oval in Fig. 6(e), suggests that even a nominally uniform film contains some small degree of anisotropy variation.

\section{C. $T_{A}=500^{\circ} \mathrm{C}$}

After annealing the graded and uniform $\mathrm{FePtCu}$ films at $500^{\circ} \mathrm{C}$, the major loops (Figs. $7($ a) and $7(\mathrm{~d})$ ), FORC

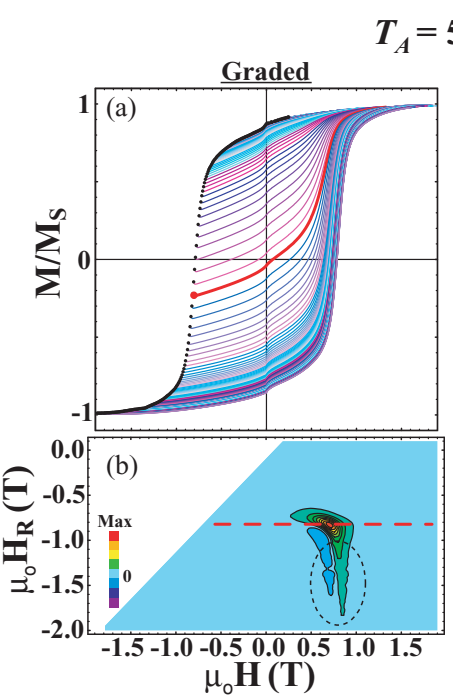

$T_{A}=500{ }^{\circ} \mathrm{C}$
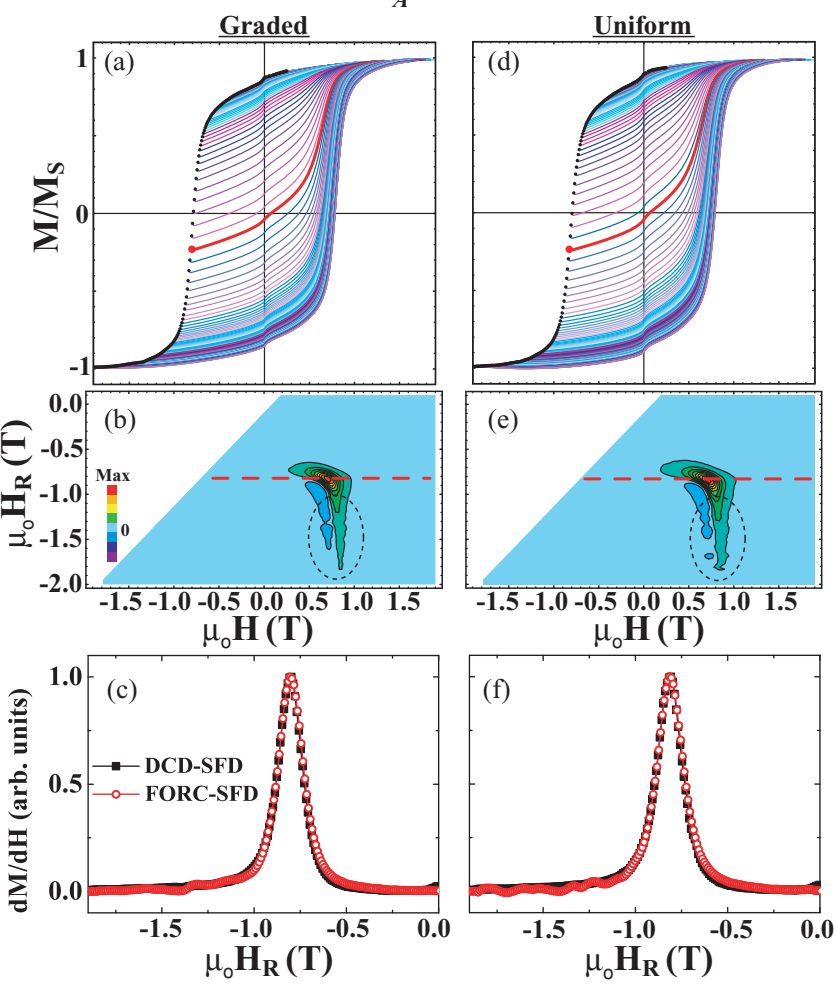

FIG. 7. (Color online) Families of FORCs, FORC distributions, and SFDs for the graded (left) and uniform (right) $\mathrm{FePtCu}$ films annealed at $500^{\circ} \mathrm{C}$. The first point of each reversal curve in (a) and (d) is shown by a black dot, and the corresponding FORC distributions, shown as contour plots, are in (b) and (e), respectively. The horizontal (red) dashed lines in (b) and (e) correspond to the (red) bold FORCs in (a) and (d), respectively. The dashed black ovals in (b) and (e) highlight regions of the FORC diagrams discussed in the text. The DCD-SFD as closed (black) squares and FORC-SFD as open (red) circles for the graded and uniform films are shown in (c) and (f), respectively.

distributions (Figs. 7(b) and 7(e)), and SFDs (Figs. 7(c) and 7(f)) become virtually indistinguishable. As discussed earlier, in regards to the PNR data, this suggests the $\mathrm{Cu}$ in the graded $\mathrm{FePtCu}$ sample has diffused throughout the film at this elevated $T_{A}$. It is also consistent that the graded and uniform samples should become identical, because both contain the same total $\mathrm{Cu}$ content. The coercivity of the major loop has further increased to $805 \mathrm{mT}$ as the high anisotropy $\mathrm{L}_{0}$ phase has become better established and more uniformly distributed through the film thickness. The FORC distributions (Figs. 7(b) and 7(e)) show a single peak followed by an irreversible tail, indicating a reversal mechanism similar to that found in the uniform FePtCu samples annealed at $300^{\circ} \mathrm{C}$ (Fig. 4(e)) and $400{ }^{\circ} \mathrm{C}$ (Fig. 6(e)). Furthermore, as was observed in the other uniform FePtCu samples, the DCD-SFD and the FORC-SFD (Figs. 7(c) and 7(f)) not only overlap but also are highly symmetric. Finally, the residual high anisotropy tail, indicated with a black dashed oval, is still present, possibly because of anisotropy variations from deposition fluctuations and/or temperature inhomogeneities during annealing. 


\section{CONCLUSIONS}

We conducted a detailed analysis of how the magnetic properties of both compositionally uniform and graded $\mathrm{FePtCu}$ films evolve as a function of $T_{A}$. PNR measurements show that $\mathrm{Cu}$-graded $\mathrm{FePtCu}$ films exhibit significant depth-dependent magnetization variations for $T_{A}$ of $300^{\circ} \mathrm{C}$ and $400^{\circ} \mathrm{C}$ that are consistent with the expected compositional gradient. After annealing at $500{ }^{\circ} \mathrm{C}$, a drastic reduction in the slope of the magnetic profile is found for the graded sample that is consistent with interdiffusion of the $\mathrm{Cu}$. In addition, all samples show a finite magnetization in the capping layer, most likely because of a fraction of Fe diffusing into the Ta. Furthermore, the field-dependent magnetization profiles clearly indicate depth-dependent magnetic anisotropy variations. Besides the very soft capping layer, the magnetic profile of the graded sample annealed at $300{ }^{\circ} \mathrm{C}$ shows that the anisotropy gradient is not well established at this low $T_{A}$, because only the very bottom of the film has transformed into the hard $\mathrm{L} 1_{0}$ phase. The FORC analysis is consistent, indicating dominant soft phase switching with a significant hard phase component to the reversal. As the $T_{A}$ is increased to $400{ }^{\circ} \mathrm{C}$, dramatic changes to the anisotropy profile and reversal mechanisms are observed. Besides the magnetic soft capping layer, the field-dependent PNR measurements now indicate that the bottom of the film is comparatively soft. Although the increased $T_{A}$ has transformed a larger portion of the film into the $\mathrm{L} 1_{0}$ phase, the significant $\mathrm{Cu}$ compositional gradient also decreases the relative anisotropy strength toward the bottom, $\mathrm{Cu}$-rich region of the film. The FORC measurements confirm that the anisotropy gradient has become better established as the gradual reversal of the soft layers are found to facilitate the switching of the entire film. The switching field is indeed smaller than the uniform reference sample annealed at the same temperature. Finally, after annealing at $500{ }^{\circ} \mathrm{C}$, the compositionally uniform and graded samples become virtually indistinguishable. This is consistent with the PNR results and suggests significant interdiffusion of the $\mathrm{Cu}$ in the compositionally graded $\mathrm{FePtCu}$ samples at this elevated temperature.

\section{ACKNOWLEDGMENTS}

Support from the Swedish Foundation for Strategic Research (SSF), the Swedish Research Council (VR), the Göran Gustafsson Foundation, and the Knut and Alice Wallenberg Foundation is gratefully acknowledged. V.B. thanks the Blanceflor Boncompagni-Ludovisi Foundation. J.N. thanks the Catalan Direcció General de Recerca (Grant No. 2009SGR1292) and the Spanish Ministerio de Ciencia e Innovación (Grant No. MAT2010-20616-C02) projects for partial financial support. We thank P. A. Kienzle of NIST for assistance with the Refl1D reflectometry modeling software, supported by NSF DMR-0520547. J.A. is a Royal Swedish Academy of Sciences Research Fellow supported by a grant from the Knut and Alice Wallenberg Foundation. *randydumas@gmail.com

${ }^{1}$ J. Nogués and I. K. Schuller, J. Magn. Magn. Mater. 192, 203 (1999).

${ }^{2}$ O. Hellwig, J. B. Kortright, K. Takano, and E. E. Fullerton, Phys. Rev. B 62, 11694 (2000).

${ }^{3}$ E. F. Kneller and R. Hawig, IEEE Trans. Magn. 27, 3588 (1991).

${ }^{4}$ D. Suess, Appl. Phys. Lett. 89, 113105 (2006).

${ }^{5}$ D. Suess, J. Fidler, G. Zimanyi, T. Schrefl, and P. Visscher, Appl. Phys. Lett. 92, 173111 (2008).

${ }^{6}$ K. Z. Gao and H. N. Bertram, IEEE Trans. Magn. 38, 3675 (2002).

${ }^{7}$ J. P. Wang, Nat. Mater. 4, 191 (2005).

${ }^{8}$ R. H. Victora and X. Shen, IEEE Trans. Magn. 41, 537 (2005).

${ }^{9}$ T. W. McDaniel, J. Phys. Condens. Matter 17, R315 (2005).

${ }^{10}$ J. G. Zhu, X. C. Zhu, and Y. H. Tang, IEEE. Trans. Magn. 44, 125 (2008).

${ }^{11}$ M. H. Kryder and R. W. Gustafson, J. Magn. Magn. Mater. 287, 449 (2005).

${ }^{12}$ H. J. Richter, IEEE Trans. Magn. 35, 2790 (1999).

${ }^{13}$ J. Lee, V. Alexandrakis, M. Fuger, B. Dymerska, D. Suess, D. Niarchos, and J. Fidler, Appl. Phys. Lett. 98, 222501 (2011).

${ }^{14}$ V. Alexandrakis, D. Niarchos, K. Mergia, J. Lee, J. Fidler, and I. Panagiotopoulos, J. Appl. Phys. 107, 013903 (2010).

${ }^{15}$ J. S. Chen, L. S. Huang, J. F. Hu, G. Ju, and G. M. Chow, J. Phys. D: Appl. Phys. 43, 185001 (2010).

${ }^{16}$ D. Goll, A. Breitling, L. Gu, P. A. van Aken, and W. Sigle, J. Appl. Phys. 104, 083903 (2008).
${ }^{17}$ B. J. Kirby, J. E. Davies, K. Liu, S. M. Watson, G. T. Zimanyi, R. D. Shull, P. A. Kienzle, and J. A. Borchers, Phys. Rev. B 81, 100405 (2010).

${ }^{18}$ B. J. Kirby, S. M. Watson, J. E. Davies, G. T. Zimanyi, K. Liu, R. D. Shull, and J. A. Borchers, J. Appl. Phys. 105, 07 C929 (2009).

${ }^{19}$ T. J. Zhou, B. C. Lim, and B. Liu, Appl. Phys. Lett. 94, 152505 (2009).

${ }^{20}$ C. L. Zha, R. K. Dumas, Y. Y. Fang, V. Bonanni, J. Nogués, and J. Åkerman, Appl. Phys. Lett. 97, 182504 (2010).

${ }^{21}$ V. Bonanni, Y. Y. Fang, R. K. Dumas, C. L. Zha, S. Bonetti, J. Nogués, and J. Åkerman, Appl. Phys. Lett. 97, 202501 (2010).

${ }^{22}$ C. L. Zha, S. Bonetti, J. Persson, Y. Zhou, and J. Åkerman, J. Appl. Phys. 105, 07E910 (2009).

${ }^{23}$ C. L. Zha, Y. Y. Fang, J. Nogués, and J. Åkerman, J. Appl. Phys. 106, 053909 (2009).

${ }^{24}$ Y. Zhou, S. Bonetti, C. L. Zha, and J. Åkerman, New J. Phys. 11, 103028 (2009).

${ }^{25}$ Y. Zhou, C. L. Zha, S. Bonetti, J. Persson, and J. Åkerman, Appl. Phys. Lett. 92, 262508 (2008).

${ }^{26}$ J. E. Davies, O. Hellwig, E. E. Fullerton, G. Denbeaux, J. B. Kortright, and K. Liu, Phys. Rev. B 70, 224434 (2004).

${ }^{27}$ J. E. Davies, O. Hellwig, E. E. Fullerton, J. S. Jiang, S. D. Bader, G. T. Zimanyi, and K. Liu, Appl. Phys. Lett. 86, 262503 (2005).

${ }^{28}$ R. K. Dumas, C.-P. Li, I. V. Roshchin, I. K. Schuller, and K. Liu, Phys. Rev. B 75, 134405 (2007).

${ }^{29}$ C. R. Pike, A. P. Roberts, and K. L. Verosub, J. Appl. Phys. 85, 6660 (1999). 
${ }^{30}$ R. K. Dumas, T. Gredig, C. P. Li, I. K. Schuller, and K. Liu, Phys. Rev. B 80, 014416 (2009).

${ }^{31}$ R. K. Dumas, K. Liu, C. P. Li, I. V. Roshchin, and I. K. Schuller, Appl. Phys. Lett. 91, 202501 (2007).

${ }^{32}$ J. E. Davies, J. Wu, C. Leighton, and K. Liu, Phys. Rev. B 72, 134419 (2005).

${ }^{33}$ J. E. Davies, O. Hellwig, E. E. Fullerton, and K. Liu, Phys. Rev. B 77, 014421 (2008).

${ }^{34}$ J.-W. Liao, R. K. Dumas, H.-C. Hou, Y.-C. Huang, W.-C. Tsai, L.-W. Wang, D.-S. Wang, M.-S. Lin, Y.-C. Wu, R.-Z. Chen, C.-H. Chiu, J. W. Lau, K. Liu, and C.-H. Lai, Phys. Rev. B 82, 014423 (2010).

${ }^{35}$ M. T. Rahman, R. K. Dumas, N. Eibagi, N. N. Shams, Y.-C. Wu, K. Liu, and C.-H. Lai, Appl. Phys. Lett. 94, 042507 (2009).

${ }^{36}$ J. Wong, P. Greene, R. K. Dumas, and K. Liu, Appl. Phys. Lett. 94, 032504 (2009).

${ }^{37}$ C. L. Zha, J. Persson, S. Bonetti, Y. Y. Fang, and J. Åkerman, Appl. Phys. Lett. 94, 163108 (2009).
${ }^{38}$ T. Maeda, T. Kai, A. Kikitsu, T. Nagase, and J.-I. Akiyama, Appl. Phys. Lett. 80, 2147 (2002).

${ }^{39}$ C. F. Majkrzak, Physica B 221, 342 (1996).

${ }^{40}$ C. F. Majkrzak, K. V. O'Donovan, and N. F. Berk, Neutron Scattering from Magnetic Materials. (Elsevier Science, New York, 2005).

${ }^{41}$ Although instrument resolution was insufficient to detect oscillations corresponding to $1 \mu \mathrm{m}$ of $\mathrm{SiO}_{2}$, this layer is assumed to be present in the model, and the resulting reflectivity calculations are sufficiently oversampled to prevent aliasing artifacts.

${ }^{42}$ M. L. Yan, Y. F. Xu, and D. J. Sellmyer, J. Appl. Phys. 99, 08G903 (2006).

${ }^{43}$ Y. K. Takahashi, M. Ohnuma, and K. Hono, J. Magn. Magn. Mater 246, 259 (2002).

${ }^{44}$ D. C. Berry and K. Barmak, J. Appl. Phys. 102, 024912 (2007).

${ }^{45}$ Y. Y. Fang, R. K. Dumas, C. L. Zha, and J. Åkerman, IEEE Magn. Lett. 2, 5500104 (2011). 